\title{
Educación, Gobierno Abierto y progreso: los Objetivos de Desarrollo Sostenible (ODS) en el ámbito educativo. Una visión crítica de la LOMLOE
}

Education, Open Government and progress: The Sustainable Development Goals (SDGs) in education. A critical view of LOMLOE

\author{
María Dolores Montero Caro \\ Profesora Sustituta Interina (Derecho Constitucional) \\ Departamento de Derecho Público y Económico \\ Facultad de Derecho y Ciencias Económicas y Empresariales \\ Universidad de Córdoba (España) \\ E-mail: $\underline{\text { mdmontero@uco.es }}$
}

\begin{abstract}
Resumen: La Agenda 2030 aprobada por Naciones Unidas en 2015, supuso un impulso a los Estados Miembros para atender a unos objetivos universales en materia de desarrollo sostenible, los conocidos como ODS. Evidentemente, y teniendo en consideración que entre los países firmantes se encuentran muchos en desarrollo, la garantía en el acceso a una educación de calidad conforma uno de los objetivos fundamentales de esta Agenda. Al respecto, España ha dado pasos importantes en la implementación de este objetivo, muestra de ello es la creación de un Ministerio específico sobre Derechos Sociales y Agenda 2030 compartido con la Vicepresidencia segunda del Gobierno. Si bien es cierto que, en lo concerniente al ámbito normativo, la reciente aprobación de la LOMLOE, también conocida como
\end{abstract}


Ley Celáa, ha sido la que ha puesto sobre la mesa cambios y medidas concretas para alcanzar las metas del ODS4.

Por otra parte, la reciente aprobación del IV Plan de Acción de Gobierno Abierto 2020-2024 incorpora entre los elementos dirigidos a construir una sociedad más democrática basada en los valores de paz, justicia e instituciones más sólidas (ODS 16), algunas iniciativas y compromisos que fomentan la participación de los más jóvenes como fundamento también de una educación de calidad.

Palabras clave: Objetivos de desarrollo sostenible (ODS); Educación, Gobierno Abierto; LOMLOE.

\begin{abstract}
The 2030 Agenda approved by the United Nations in 2015 was an impetus for Member States to meet universal sustainable development goals, known as SDGs. Evidently, and taking into consideration that many of the signatory countries are developing countries, guaranteeing access to quality education is one of the fundamental objectives of this Agenda. In this regard, Spain has taken important steps in the implementation of this goal, as evidenced by the creation of a specific Ministry for Social Rights and Agenda 2030, shared with the Second VicePresidency of the Government. Although it is true that, in terms of legislation, it is the recent approval of the LOMLOE, also known as the Celáa Law, that has brought changes and concrete measures to the table to achieve the SDG4 targets.

Moreover, the recent approval of the IV Open Government Action Plan 2020-2024 incorporates, among the elements aimed at building a more democratic society based on the values of peace, justice and stronger institutions (SDG 16), some initiatives and commitments that encourage the participation of young people as a foundation for quality education.
\end{abstract}

Keywords: Sustainable development goals (SDGs); Education; Open Government; LOMLOE. 


\section{Introducción}

La educación es un elemento sobre el que vertebrar la estructura democrática de las organizaciones sociales. Ello no es desconocido para los actores políticos por lo que «la educación ha sido uno de los campos de batalla donde librar debates políticos generales, especialmente disputas religiosas»(Cotino, 2012: 9).

Conseguir una educación de calidad, además de ocupar un objetivo específico dentro de la Agenda 2030, es, sin duda, una prioridad para cualquier sistema democrático actual. No hay que perder de vista que, «además de ser un fin en sí misma, la educación se considera un instrumento clave para el logro de todos los ODS» (Gamba y Arias, 2017: 14). No obstante, la calificación «de calidad» puede ser interpretada de diversas formas dependiendo de la sociedad, país, o incluso, de la ideología política que presente el gobierno encargado de gestionar la normativa educativa en un ámbito temporal y territorial concreto.

Así, se trata de un ámbito que no ha pasado desapercibido para la doctrina, especialmente atenta a todas las vicisitudes en las que se ha ido encontrando el sistema educativo. Obviamente, desde la perspectiva actual, el COVID-19 tiene un papel protagonista en todos los aspectos sociales, por supuesto, también, en la esfera educativa. Ello no es óbice para subrayar las diferentes situaciones en las que se han tomado opciones políticas diversas para acomodar un mejor y más amplio avance educativo de la sociedad y, con ello, la consiguiente función legislativa de los Estados.

Desde que en 2018 se aprobase el Plan de Acción para la implementación de la Agenda 2030 en España, donde se definieron algunas de las llamadas políticas palanca para los Objetivos de Desarrollo Sostenible (ODS, en adelante), son diversas las acciones que ya se han materializado en forma de acuerdos, reformas o aprobación de nuevas normas. En este sentido, tras la aprobación en 2015 de la Agenda 2030 sobre desarrollo sostenible y, a una década de que cumpla el plazo para la implementación de los objetivos a los que se comprometieron los Estados Miembros de las Naciones Unidas, España ha dado pequeños avances en el 
cumplimento del ODS 4: «Garantizar una educación inclusiva, equitativa y de calidad y promover oportunidades de aprendizaje durante toda la vida para todos».

Entre las acciones que ya se han llevado a cabo o que están previstas para el futuro inmediato, sin duda, la aprobación de la Ley Orgánica 3/2020, de 29 de diciembre, por la que se modifica la Ley Orgánica 2/2006, de 3 de mayo, de Educación, puede entenderse como el buque insignia de las medidas tendentes a cumplir con el ODS 4, si bien, tal y como se expondrá en los epígrafes subsiguientes, no será la única.

\section{El derecho constitucional a la educación y su desarrollo normativo.}

Son numerosos los estudios y obras que tratan de forma pormenorizada el derecho a la educación enfatizando en cada una de sus aristas: como derecho humano, como derecho fundamental, su regulación internacional y nacional, entre otras cuestiones que dependerán del enfoque particular de la disciplina jurídica que trate el asunto. Dentro de este marco y, teniendo presente que no se trata del eje principal de este estudio, sí que es necesario realizar un breve esbozo de la regulación del derecho a la educación en España y su desarrollo normativo hasta llegar a la actual aprobación de la Ley Orgánica 3/2020, de 29 de diciembre, por la que se modifica la Ley Orgánica 2/2006, de 3 de mayo, de Educación (LOMLOE, en adelante).

Desde el punto de vista internacional y, que claramente ha servido de inspiración para la redacción del artículo 27 de la Constitución Española (CE, en adelante), encontramos el artículo 26 de la Declaración Universal de Derechos Humanos (DUDH, en adelante) de 1948 que establece una diferenciación entre los niveles de educación, garantizando la gratuidad y obligatoriedad de la educación elemental y dejando para los estudios superiores el acceso en condiciones de igualdad y en función de los requisitos de mérito. Asimismo, en su apartado tercero se recoge el derecho preferente de los padres a escoger el tipo de educación que habrá de darse a sus hijos. Siguiendo con el recorrido por los Tratados internacionales que han reconocido el alcance del derecho a la educación es inevitable señalar los artículos 13 y 14 del Pacto Internacional de Derechos Económicos, Sociales y Culturales (PIDESC, en adelante) de 1966. Ambos preceptos, además de incluir los postulados 
de la DUDH, precisa aún más las características de obligatoriedad y gratuidad de cada una de las fases, el carácter prestacional del derecho por parte de los Estados, así como la libertad de los padres en la elección de la educación para sus hijos, en especial, la concerniente al ámbito religioso o moral de acuerdo con sus propias convicciones. Este último aspecto es regulado con mayor detalle en el apartado cuarto del artículo 18 del Pacto Internacional de Derechos Civiles y Políticos (PIDCP, en adelante), aprobado también en 1966 por la Asamblea de las Naciones Unidas cuando, al referirse a la libertad de pensamiento, conciencia y religión establece que:

Art. 18.4 PIDCP. «Los Estados Parte en el presente Pacto se comprometen a respetar la libertad de los padres y, en su caso, de los tutores legales, para garantizar que los hijos reciban la educación religiosa y moral que esté de acuerdo con sus propias convicciones».

Todas estas manifestaciones se ven complementadas con las referencias del derecho a la educación en la Convención sobre Derechos del Niño (CDN, en adelante) de 1989 (en concreto en sus artículos 28 y 29) o en la Convención relativa a la lucha contra la discriminación en la esfera de la enseñanza de 1960.

En lo concerniente al ámbito europeo, la Carta de Derechos Fundamentales de la Unión Europea proclama el derecho a la educación en su artículo 14 en sintonía con lo ya recogido en los textos internacionales. Una mayor labor de precisión la encontramos, por ejemplo, en la Carta Europea de Derechos del Niño, en la que se reconoce de forma amplia y pormenorizada el contenido del derecho a la educación de los niños, así como el carácter prestacional que hace posible que una situación económica desfavorable de los padres no suponga un límite para la efectividad de este derecho.

Descendiendo ya al ámbito estatal, siendo éste el cimiento del presente estudio, la base de la configuración del derecho a la educación en España se localiza en el artículo 27 CE. En síntesis, nos encontramos ante un derecho subjetivo, de carácter 
prestacional y que además reconoce también un derecho de libertad, como es la libertad de enseñanza.

El hecho de que el artículo 27 CE sea el más extenso de todos los que componen el Título I es un ejemplo evidente de no sólo su importancia como derecho fundamental, sino también del amplio y diverso contenido básico que engloba.

La vinculación de este derecho con el libre desarrollo de la personalidad, consagrado en el artículo 10 de nuestro texto constitucional, es innegable. De este modo, el derecho a la educación se entiende como una de las manifestaciones más claras del desarrollo de la personalidad del individuo fundamento del orden jurídico y la paz social y, por ende, vinculado al concepto de dignidad humana.

No cabe duda de que la educación de los niños, así como la educación superior, trasciende del mero desarrollo individual del estudiante. En efecto, como miembros de una sociedad, sus resultados se expanden al ámbito de la colectividad, sirviendo también para «la consolidación democrática y eficacia misma de los derechos fundamentales» (Cotino, 2012: 84). En esta línea se ha pronunciado en innumerables ocasiones Naciones Unidas vinculando la educación con la igualdad de género, con la lucha contra el racismo, con la resolución pacífica de conflictos, con la protección del medio ambiente o con la participación en la esfera pública, entre otros aspectos, cuya finalidad principal es la de construir sociedades democráticas responsables ${ }^{1}$. La aprobación de la Agenda 2030 sobre desarrollo sostenible en el año 2015 aparece, por tanto, como una recopilación de todos los objetivos y metas para alcanzar sociedades más responsables y democráticas que ya habían sido previamente pronunciados por la propia Asamblea de Naciones Unidas desde su creación en diferentes ocasiones.

\footnotetext{
${ }^{1}$ Entre los pronunciamientos destacados de Naciones Unidas, previos a la aprobación de la Agenda 2030, sobre el alcance real del derecho a la educación destacan: El Comité de Derechos del Niño 2001, concretamente Observación General núm. 1: Propósitos de la educación; la Resolución 57/254 de 2002 relativa al decenio de las Naciones Unidas de la Educación para el Desarrollo Sostenible (2005-2014) o el Programa de Acción sobre una Cultura de Paz aprobada por la Asamblea el 13 de septiembre de 1999 por la que se declaraba la Década Internacional para la Cultura de Paz (20012010).
} 
Dentro de este marco, desde la aprobación de la Constitución en 1978, en España se han sucedido diferentes leyes orgánicas reguladoras de este derecho que, junto con las normas de rango inferior y las normas educativas a nivel autonómico, han venido conformando un amplio desarrollo normativo. Las normas reguladoras del derecho a la educación se han visto alteradas de forma considerable, coincidiendo con el cambio de color político del gobierno, lo cual no deja de ser una muestra más de la importancia que presenta la educación y la enseñanza de valores como instrumento para el desarrollo de la personalidad individual con proyección colectiva.

En la siguiente tabla se muestra una cronología de todas las leyes orgánicas reguladoras del derecho a la educación aprobadas en nuestro país en período democrático.

Tabla 1: Cronología de las leyes educativas estatales aprobadas desde la entrada en vigor de la CE.

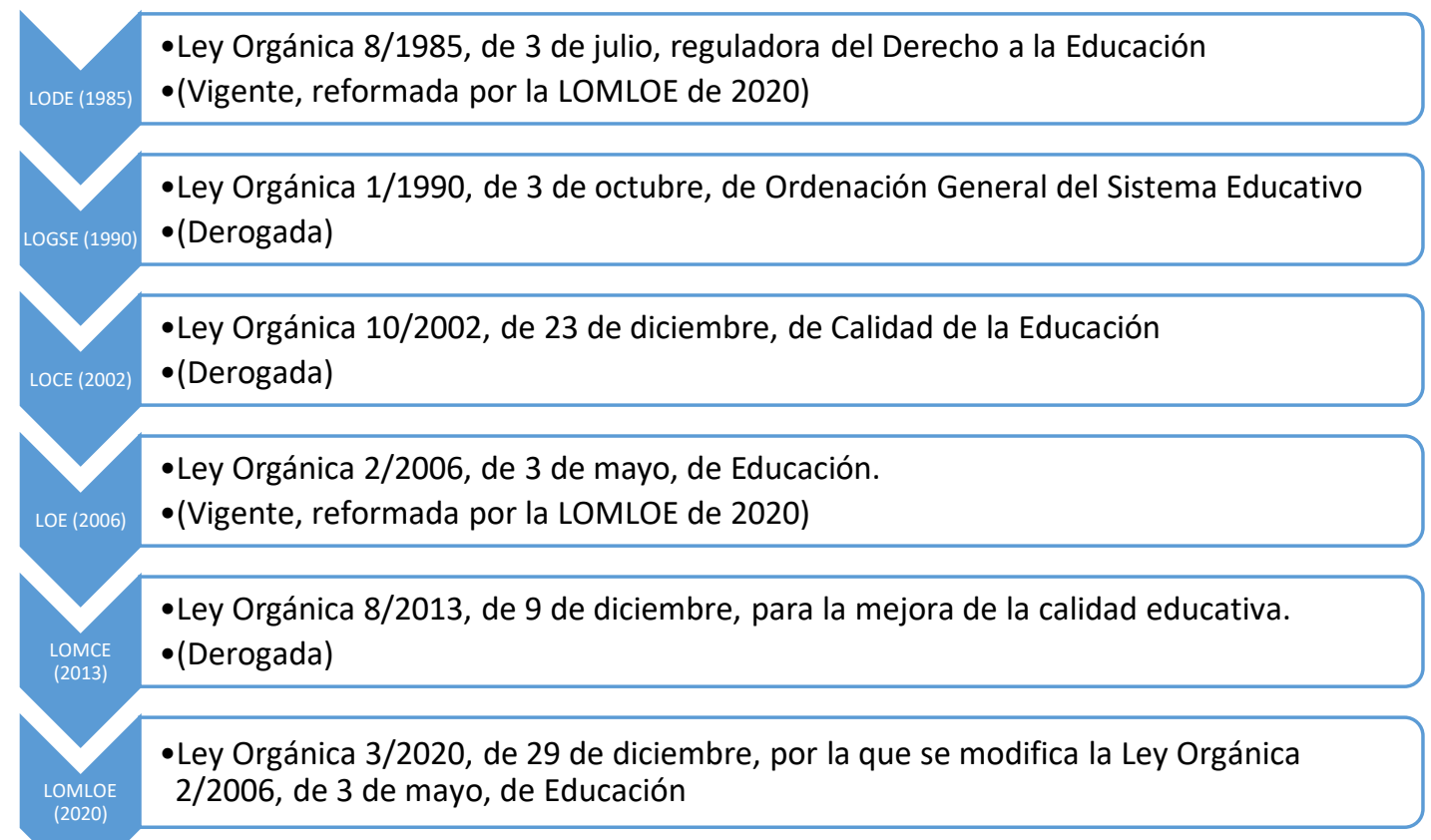




\section{Agenda 2030 y derecho a la educación.}

Una vez presentadas, de forma esquemática, las diversas normas reguladoras del derecho a la educación aprobadas en nuestro país, conviene afirmar que, a pesar de las preferencias políticas, existen una serie de objetivos y retos que deben ser universales en esta materia. En esta línea se pronuncia Vidal cuando afirma que «en España hay retos evidentes que merecen el esfuerzo conjunto: seguir disminuyendo los todavía altos índices de abandono escolar, mejorar la calidad de nuestro sistema educativo, seguir garantizando la igualdad y la equidad en el acceso a la educación...» $(2017,128)$.

Si bien, tras más de cuarenta años de democracia no hemos sido capaces de aprobar un Pacto de Estado por la educación, evitando así la incertidumbre de los constantes cambios normativos, desde Naciones Unidas sí que han puesto todos sus esfuerzos para que los países, tanto desarrollados como en vías de desarrollo, emprendan un nuevo camino que permita mejorar la vida de todos, sin dejar a nadie atrás, siendo la educación uno de los objetivos prioritarios para ello. Es así como surgió en septiembre de 2015 la Agenda 2030 para el Desarrollo Sostenible, conocida también simplemente como Agenda 2030.

Dentro de este marco, los Estados Miembros de las Naciones Unidas que aprobaron la Agenda 2030 se comprometieron a alcanzar 17 Objetivos de Desarrollo Sostenible en un plazo de 15 años, es decir, en 2030, de ahí su denominación. Cabe apuntar que, como indican Martínez Usarralde y Lloret «en la Agenda 2030 se otorga más relevancia a cuestiones como el aprendizaje a lo largo de la vida, la equidad, la igualdad de género y la inclusión de los sistemas educativo» (2020: 341). 


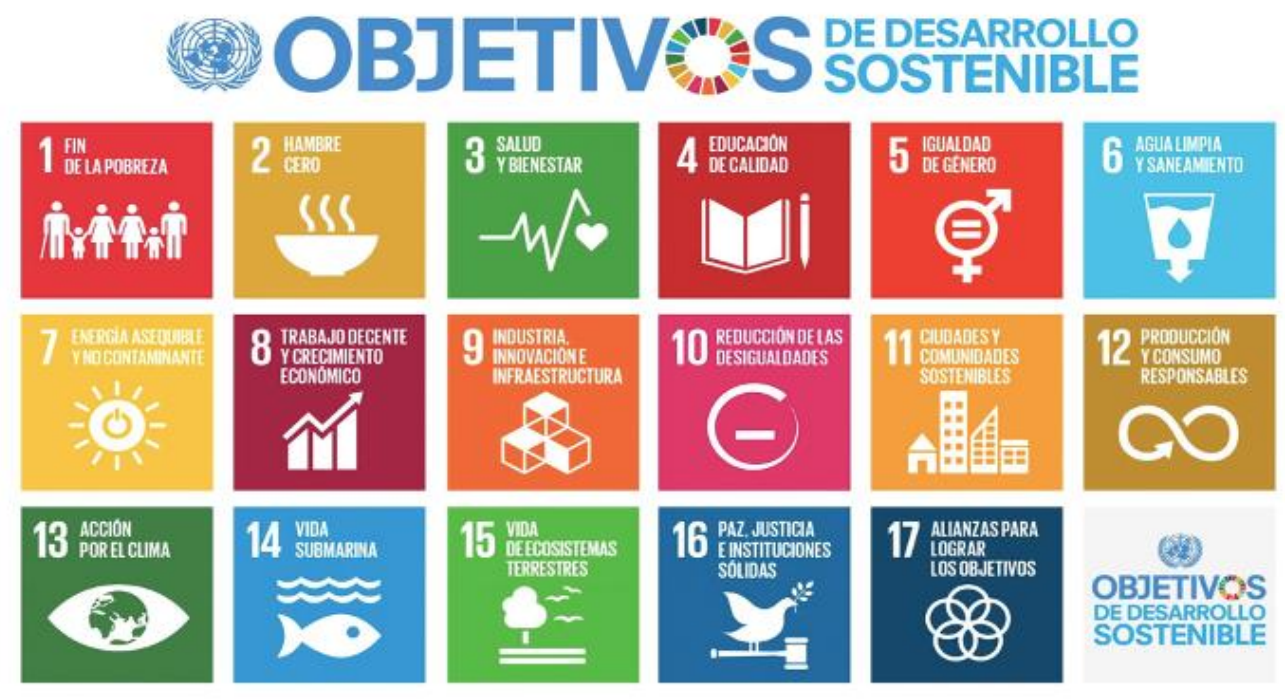

Fuente: Web Naciones Unidas. Agenda 2030. Disponible en:

https://www.un.org/sustainabledevelopment/es/objetivos-de-desarrollo-sosteniblel

Tal y como se infiere de la ilustración anterior, existe un objetivo particular que hace referencia a la educación, el ODS 4: Garantizar una educación inclusiva, equitativa y de calidad y promover oportunidades de aprendizaje durante toda la vida para todos. Al igual que los otros $16 \mathrm{ODS}$, este objetivo se compone de una serie de metas concretas previstas para que en 2030 se consiga la tan ansiada educación de calidad.

Tabla 1: metas que componen el ODS 4

4.1 De aquí a 2030, asegurar que todas las niñas y todos los niños terminen la enseñanza primaria y secundaria, que ha de ser gratuita, equitativa y de calidad y producir resultados de aprendizaje pertinentes y efectivos

4.2 De aquí a 2030, asegurar que todas las niñas y todos los niños tengan acceso a servicios de atención y desarrollo en la primera infancia y educación preescolar de calidad, a fin de que estén preparados para la enseñanza primaria

4.3 De aquí a 2030, asegurar el acceso igualitario de todos los hombres y las mujeres a una formación técnica, profesional y superior de calidad, incluida la enseñanza universitaria

4.4 De aquí a 2030, aumentar considerablemente el número de jóvenes y adultos que tienen las competencias necesarias, en particular técnicas y profesionales, para acceder al empleo, el trabajo decente $y$ el emprendimiento 
4.5 De aquí a 2030, eliminar las disparidades de género en la educación y asegurar el acceso igualitario a todos los niveles de la enseñanza y la formación profesional para las personas vulnerables, incluidas las personas con discapacidad, los pueblos indígenas y los niños en situaciones de vulnerabilidad

4.6 De aquí a 2030, asegurar que todos los jóvenes y una proporción considerable de los adultos, tanto hombres como mujeres, estén alfabetizados y tengan nociones elementales de aritmética

4.7 De aquí a 2030, asegurar que todos los alumnos adquieran los conocimientos teóricos y prácticos necesarios para promover el desarrollo sostenible, entre otras cosas mediante la educación para el desarrollo sostenible y los estilos de vida sostenibles, los derechos humanos, la igualdad de género, la promoción de una cultura de paz y no violencia, la ciudadanía mundial y la valoración de la diversidad cultural y la contribución de la cultura al desarrollo sostenible

4.a Construir y adecuar instalaciones educativas que tengan en cuenta las necesidades de los niños y las personas con discapacidad y las diferencias de género, y que ofrezcan entornos de aprendizaje seguros, no violentos, inclusivos y eficaces para todos

4.b De aquí a 2020, aumentar considerablemente a nivel mundial el número de becas disponibles para los países en desarrollo, en particular los países menos adelantados, los pequeños Estados insulares en desarrollo y los países africanos, a fin de que sus estudiantes puedan matricularse en programas de enseñanza superior, incluidos programas de formación profesional y programas técnicos, científicos, de ingeniería y de tecnología de la información y las comunicaciones, de países desarrollados y otros países en desarrollo

De aquí a 2030, aumentar considerablemente la oferta de docentes calificados, incluso mediante la cooperación internacional para la formación de docentes en los países en desarrollo, especialmente los países menos adelantados y los pequeños Estados insulares en desarrollo

Fuente: https://www.un.org/sustainabledevelopment/es/education/

Si bien, los ODS tienen como clave temporal el año 2030, es importante destacar que la propia ONU adelantó el plazo de logro al año 2020 en 21 de las 169 metas que integran los ODS. Tal es el caso, como se puede ver en la tabla 1, de la meta 4.b referente a la necesidad de aumentar considerablemente el número de becas en los países en desarrollo, en particular en los pequeños Estados insulares en desarrollo y en los países africanos, para así poder optar a programas de enseñanza superior. Dentro de este marco, una vez superado el año 2020, Naciones Unidas en su Informe Anual sobre los ODS de 2020 ha afirmado que, si bien han existido progresos, estos han sido insuficientes para alcanzar la meta 4.b. En efecto, hubo un ligero aumento 
en la asistencia oficial para el desarrollo en becas con respecto al año 2017, siendo las principales regiones receptoras Asia y África, y los principales países beneficiarios Filipinas, Indonesia, la República de Moldavia y Vietnam.

Una vez superado el año 2020, teniendo presente que quedan menos de diez años para alcanzar los ODS que componen la Agenda 2030, en la cumbre de septiembre de 2019 se acordó un decenio de acción con la finalidad de poner todos los esfuerzos en movilizar la financiación, mejorar la aplicación a nivel nacional y fomentar la resiliencia, así como reforzar las instituciones para cumplir los Objetivos en la fecha prevista, el año 2030, sin dejar a nadie atrás.

Sin embargo, a pesar de la buena intención por acelerar la acción a todos los niveles con el acuerdo del decenio de acción, la llegada y posterior expansión de la pandemia provocada por el COVID-19 en 2020 ha puesto de relieve la paralización de muchas de las metas de los ODS. En este sentido, en el ámbito educativo el ejemplo más palpable lo encontramos en el cierre de las escuelas que ha afectado de forma negativa en los resultados de la enseñanza y desarrollo de los niños y jóvenes, provocando una brecha de desigualdad mayor en lugares donde no ha sido posible el aprendizaje a distancia.

En España, el Informe de Progreso de 2020 «Reconstruir lo común. La implementación de la Agenda 2030», realiza un análisis pormenorizado de las medidas ejecutadas en nuestro país tendentes a cumplir los ODS, así como aquellas otras previstas en el corto plazo. Como no podía ser de otro modo, la aprobación del Real Decreto 463/2020, de 14 de marzo, por el que se declaró el estado de alarma para la gestión de la situación de crisis sanitaria ocasionada por el COVID19, y sus consecuencias sociales, copa la mayor parte de este informe. Asimismo, no podemos olvidar que España es un país profundamente descentralizado lo cual contribuye a que buena parte del logro de los ODS se desarrollen en el nivel local y autonómico. De hecho, la mayoría de las comunidades y ciudades autónomas han elaborado y aprobado estrategias para la implementación de la Agenda 2030. 
Centrándonos en el ámbito que nos ocupa en este estudio, es decir, el ODS 4, la propuesta del grupo de trabajo de la comisión permanente del Consejo de Desarrollo Sostenible para la Agenda 2030, reunido en enero de 2021, plantea diversos retos para avanzar en el cumplimiento de éste y otros objetivos. En concreto, el reto «sistema educativo universal, de calidad y basado en la equidad», pretende reducir las desigualdades educativas ocasionadas por situaciones socioeconómicas desfavorables o discriminación por pertenencia a un determinado grupo étnico-racial o por tener alguna discapacidad. Para ello proponen siete políticas transformadoras que da la impresión de tratarse de meras declaraciones de intenciones. Muchas de ellas, como el ya comentado infructífero Pacto de Estado por la Educación, o la escolarización universal entre los 0-3 años, son ideas recogidas en numerosos programas electorales en los últimos años sin que haya llegado a materializarse hasta ahora. Sin duda, la intención del ejecutivo en materia educativa para conseguir la tan ansiada educación de calidad es loable, no obstante, al tratarse de medidas a cumplir en el corto y medio plazo requieren no sólo una voluntad política del ejecutivo, sino un compromiso entre las diferentes fuerzas políticas que componen el arco parlamentario.

Políticas transformadoras insertas en el reto correspondiente al sistema educativo universal, de calidad y basado en la equidad.

\begin{tabular}{|c|c|}
\hline $\begin{array}{c}\text { ODS } \\
\text { implicado }\end{array}$ & Política transformadora \\
\hline $\begin{array}{c}\text { ODS } 4 \\
\text { ODS } 10\end{array}$ & Escolarización Universal 0-3 años \\
\hline $\begin{array}{l}\text { ODS } 4 \\
\text { ODS } 8 \\
\text { ODS } 10\end{array}$ & $\begin{array}{l}\text { Disminución de la brecha digital y de seguridad en todas sus dimensiones, } \\
\text { con atención a violencias online por razón de género y edad y formación en } \\
\text { competencia. }\end{array}$ \\
\hline ODS 4 & $\begin{array}{l}\text { Desplegar las medidas necesarias para la inclusión del alumnado con } \\
\text { discapacidad, y asegurar que se cumple la Ley de Accesibilidad en los } \\
\text { centros educativos. }\end{array}$ \\
\hline $\begin{array}{c}\text { ODS } 4 \\
\text { ODS } 8 \\
\text { ODS } 10 \\
\text { ODS } 16\end{array}$ & $\begin{array}{l}\text { Pacto de Estado/ Acuerdo de sociedad por la educación que afronte la } \\
\text { disminución del fracaso escolar; la educación individualizada; que aborde } \\
\text { el problema de segregación escolar; un sistema de becas y ayudas al estudio } \\
\text { robusto y financiado; formación y especialización del profesorado; mayor } \\
\text { calidad en la formación profesional, con especial atención a la FP Dual. }\end{array}$ \\
\hline ODS 4 & Promover la participación infantil y familiar y mejorar el clima escolar \\
\hline
\end{tabular}




\begin{tabular}{|c|l|}
\hline ODS 16 & \\
\hline ODS 4 & $\begin{array}{l}\text { Introducir en el sistema formal la Educación por la Igualdad y la Ciudadanía } \\
\text { ODS 10 }\end{array}$ \\
ODS 16 & $\begin{array}{l}\text { Otobal } \\
\text { formación para el empleo en torno a los ODS }\end{array}$ \\
\hline ODS 8 & \\
\hline
\end{tabular}

Fuente: Elaboración propia a partir de los datos del Documento del grupo de trabajo de la comisión permanente del Consejo de Desarrollo sostenible para la Agenda 2030. Disponible en: https://www.agenda2030.gob.es/recursos/docs/GTEstrategia_Retos-pais_CDS.pdf

\section{La implementación de los ODS en la nueva Ley Orgánica 3/2020, de 29 de diciembre, por la que se modifica la Ley Orgánica de Educación de 2006. (LOMLOE).}

Sin duda, hasta la fecha, la medida aprobada con más trascendencia en referencia al ODS 4 es la reciente aprobación de la Ley Orgánica 3/2020, de 29 de diciembre, por la que se modifica la Ley Orgánica de Educación de 2006.

Nos encontramos ante una norma no de contenido, sino de modificación de otras leyes existentes, de ahí su denominación como ley modificadora de la LOE de 2006. Si bien es cierto que la reforma de la LOE supone gran parte del contenido de esta nueva ley educativa, no es tampoco baladí que su entrada en vigor implica igualmente la derogación de la Ley Orgánica de Mejora de la Calidad Educativa (LOMCE) de 2013. Con la LOMCE, se marcaba «una línea clara que daba protagonismo a las familias en la educación de los hijos, frente al papel del Estado defendido, por ejemplo, en la LOE, y de nuevo en la actualidad» (Vázquez y Porto, 2020: 119).

Intereses políticos aparte, pues no podemos olvidar que la LOMCE de 2013 (también conocida como Ley Wert, en alusión al Ministro de Educación competente en esa fecha) fue impulsada por un gobierno del Partido Popular, la nueva LOMLOE (a la que también se alude a la Ministra de Educación para su denominación más informal, la Ley Celaá) critica con dureza la LOMCE en el propio Preámbulo, calificándola como una ley que «representó la ruptura del equilibrio que se había alcanzado en la 
LOE entre diferentes visiones y planteamientos acerca de la educación que deben necesariamente convivir en un sistema democrático y plural».

La LOMLOE es una ley de artículo único que incluye noventa y nueve apartados, en los que se modifica parcialmente o se da una nueva redacción a preceptos de la LOE. A este único y extenso artículo le acompañan once disposiciones adicionales, cinco transitorias, una derogatoria y seis finales.

La integración de los objetivos de la Agenda 2030, y en especial del ODS 4, es palpable tanto en el Preámbulo como en alguno de los cambios más significativos que se producen en la nueva Ley de Educación. De hecho, los cinco enfoques básicos en los que afirma asentarse la nueva ley ya nos muestran una evidencia del interés en construir una educación acorde a los principios de desarrollo sostenible marcados por la ONU. Estos enfoques básicos hacen referencia a:

- los derechos de la infancia entre los principios rectores del sistema, según lo establecido en la Convención de los Derechos del Niño de 1989;

- la igualdad de género a través de la coeducación y el fomento del aprendizaje de la igualdad efectiva entre mujeres y hombres y la prevención de la violencia de género;

- una mayor personalización del aprendizaje;

- una educación para el desarrollo sostenible y la ciudadanía mundial, así como el desarrollo de la competencia digital.

Al tratarse España de un país desarrollado, algunas de las metas que integran el ODS 4 estarían, por suerte, superadas. Por lo que el legislador se ha centrado en avanzar hacia la consecución de las metas 4.5, 4.7 y 4.a. 
Meta 4.5. De aquí a 2030, eliminar las disparidades de género en la educación y asegurar el acceso igualitario a todos los niveles de la enseñanza y la formación profesional para las personas vulnerables, incluidas las personas con discapacidad, los pueblos indígenas y los niños en situaciones de vulnerabilidad.

La perspectiva de género es, sin lugar a duda, uno de los objetivos principales que impregna la LOMLOE, no sólo en lo referente a la inclusión como materia en el currículo en las distintas etapas, sino de forma transversal en el sistema educativo en general. Un ejemplo significativo de esta particularidad lo vemos reflejado en las modificaciones realizadas al texto de la Ley Orgánica 8/1985, de 3 de julio, reguladora del Derecho de Educación (LODE) para incluir el lenguaje inclusivo (padres y madres, hijos e hijas, alumno y alumna, etc.), así como el fomento de una presencia equilibrada de mujeres y hombres en la composición del consejo escolar.

Por otro lado, el número de mujeres que acceden a titulaciones del ámbito de las ciencias, la tecnología, la ingeniería o las matemáticas sigue siendo muy inferior al de hombres. Por este motivo, la LOMCE hace un llamamiento a las Administraciones educativas a impulsar el incremento de la presencia de alumnas en estos ámbitos tradicionalmente ocupados por hombres ${ }^{2}$. Del mismo modo, se impulsará la presencia de alumnado masculino en aquellos estudios en los que exista de forma notoria una mayor matrícula de mujeres que de hombres.

Meta 4.7. De aquí a 2030, asegurar que todos los alumnos adquieran los conocimientos teóricos y prácticos necesarios para promover el desarrollo sostenible, entre otras cosas mediante la educación para el desarrollo sostenible y los estilos de vida sostenibles, los derechos humanos, la igualdad de género, la

\footnotetext{
${ }^{2}$ En el informe del Ministerio de Educación «Igualdad en Cifras MEPF 2020» se pone en evidencia la baja presencia femenina en las disciplinas de ciencias, tecnología, ingeniería y matemáticas (las conocidas como carreras STEM, por sus siglas en inglés). En este sentido, en los grados y másteres universitarios hay pocas mujeres matriculadas en carreras relacionadas con Ingeniería, industria y construcción $(28,5 \%)$ o en Informática $(12,9 \%)$, mientras que superan con creces el porcentaje con respecto a los hombres en la elección de carreras vinculadas a la Educación $(77,7 \%)$ o la Salud y Servicios Sociales $(71,4 \quad \%)$. Informe disponible en: https://www.educacionyfp.gob.es/dam/jcr:914e956e-9241-49c5-b9a6-d99d6eade751/igualdad-encifras-2020-online.pdf (consulta: enero 2021).
} 
promoción de una cultura de paz y no violencia, la ciudadanía mundial y la valoración de la diversidad cultural y la contribución de la cultura al desarrollo sostenible.

El fomento de valores democráticos y la cultura del desarrollo sostenible son elementos que se encuentran de forma reiterada tanto en el Preámbulo de la norma, como en una amplia variedad de artículos que modifica de la LOE. Al respecto, en la etapa primaria se añade la impartición en alguno de los cursos del tercer ciclo $\left(5^{\circ}\right.$ y $6^{\circ}$ de primaria) de la educación en valores cívicos y éticos. El contenido de esta materia abordará el conocimiento de la Constitución Española y el respeto de los Derechos Humanos y de la Infancia, la educación para el desarrollo sostenible y la ciudadanía mundial, la igualdad entre hombres y. mujeres, el respeto a la diversidad, el valor social de los impuestos, el fomento del espíritu crítico y la cultura de paz y no violencia (nueva redacción art. 18.3 de la LOE). Sin duda alguna, si atendemos a su literalidad, este nuevo párrafo de la LOE es claramente un reflejo de la meta 4.7 del ODS 4.

Algo similar está previsto también para la etapa de enseñanza secundaria obligatoria, pero esta vez de forma más categórica, como una asignatura obligatoria a cursar en algún curso de esta etapa por todo el alumnado. Esta asignatura, denominada «Educación en valores cívicos y éticos» tendrá el mismo contenido genérico que el ya enunciado supra para la educación primaria, pero acorde a la edad de los estudiantes. En este sentido, al cursarse esta asignatura durante la adolescencia se podrán plantear debates en profundidad en los que se subraye el importante papel individual que todas las personas tenemos como miembros de una sociedad democrática, ya que poseen una madurez cognitiva que les permite pensar de forma abstracta y resolver conflictos de un modo mucho más crítico.

Por otro lado, la LOMLOE aumenta los mecanismos participativos de la comunidad educativa a través del Consejo Escolar, a la vez que reestablece la situación anterior a la LOMCE en lo referente a la atribución de funciones correspondientes a la dirección y al Consejo Escolar (en el que también se indica expresamente que se deberá promover su presencia equilibrada de mujeres y hombres). Asimismo, en lo 
que atañe a la figura del director o directora del Centro se endurecen los requisitos para ocupar el puesto (necesidad de superar una formación específica) y se apuesta por una mayor participación del profesorado y otros miembros del Consejo Escolar en su elección. Participación que se expande también al alumnado como parte de su proceso de formación.

Sin duda, como ya se ha indicado con anterioridad, el fomento de la igualdad efectiva entre hombres y mujeres está presente a lo largo de toda la reforma planteada por la LOMLOE. Precisamente, para constatar esta idea, la nueva redacción de la Disposición adicional vigesimoquinta, mucho más amplia y pormenorizada, da cuenta de la importancia de este valor democrático en el ámbito educativo.

Meta 4.a. Construir y adecuar instalaciones educativas que tengan en cuenta las necesidades de los niños y las personas con discapacidad y las diferencias de género, y que ofrezcan entornos de aprendizaje seguros, no violentos, inclusivos y eficaces para todos.

Precisamente, junto con las modificaciones en materia de enseñanza concertada, el debate sobre la nueva configuración de la educación especial ha sido uno de los temas más polémicos desde la aprobación del proyecto de ley ${ }^{3}$. En concreto, la Disposición adicional cuarta de la nueva norma establece que, en el plazo de diez años, las Administraciones educativas deberán desarrollar un plan para que los centros ordinarios cuenten con los recursos necesarios para poder atender en las mejoras condiciones al alumnado con discapacidad, dejando así los centros de educación especial para los supuestos de atención muy especializada, convirtiéndose en centros de referencia y apoyo para los centros ordinarios. Se pretende, por tanto, la inclusión del alumnado con necesidades educativas especiales en los centros ordinarios,

\footnotetext{
${ }^{3}$ Un análisis detallado sobre esta polémica suscitada sobre la educación especial inclusiva podemos consultarla en el artículo publicado por The Conversation el 29 de noviembre de 2020, bajo el título «¿Avanza la 'ley Celaá' hacia un modelo inclusivo para el alumnado de educación especial?», de las autoras Berasategui Sancho y Darretxe Urritxi, disponible en: https://theconversation.com/avanza-laley-celaa-hacia-un-modelo-inclusivo-para-el-alumnado-de-educacion-especial-150985 (fecha de consulta: enero 2021)
} 
dejando la escolarización en centros de educación especial para los casos más complejos.

El debate está servido, pues mientras muchos padres y madres de hijos con necesidades educativas especiales consideran que se está atacando a su libertad de elección de centro, el gobierno, por su parte, entiende con esta reforma que la escolarización en centros ordinarios favorece su integración. Quizás la pregunta al respecto debería ser si los centros ordinarios están preparados para asumir necesidades educativas especiales y se deba poner el foco en desarrollar «escuelas ordinarias, en las que la diversidad sea un valor, contando con los recursos necesarios y caminando junto al saber y experiencia de profesionales que llevan muchos años respondiendo al alumnado con discapacidad» (Berasategui y Darretxe, 2020).

Del mismo modo, se incluye un nuevo artículo 20.bis que hace referencia a la especial atención a las diferencias individuales que se diagnostiquen en la etapa primaria, para poder responder con mecanismos de apoyo y refuerzo que eviten la repetición escolar, particularmente en entornos socialmente desfavorecidos. Asimismo, precisamente con el objetivo de lograr entornos de aprendizajes seguros y eficaces, en estos ámbitos se emplaza a las Administraciones a ajustar, si fuera necesario, las ratios alumno/unidad para favorecer así las estrategias pedagógicas.

En síntesis, son numerosas las alusiones de la LOMLOE a las metas que componen el ODS 4. No obstante, además de lo expuesto, la propia ley, para no dejar lugar a dudas de su claro compromiso con los ODS, incluye dos disposiciones adicionales que hacen una mención expresa a la Agenda 2030.

Disposición adicional quinta. Prioridades en los Programas de cooperación territorial. "A fin de alcanzar las metas del objetivo 4 de la Agenda 2030, el Gobierno, en colaboración con las Administraciones educativas, promoverá durante el periodo de implantación de esta Ley los programas de cooperación territorial como línea estratégica de actuación, con especial atención a mejorar los niveles de escolarización accesible y asequible en el primer ciclo de educación infantil y en formación profesional, 
así como para promover el desarrollo de las competencias, la educación inclusiva, la prevención y reducción del abandono temprano de la educación y la formación, el plurilingüismo, el fortalecimiento de la escuela rural e insular y el desarrollo profesional docente».

Disposición adicional sexta. Educación para el desarrollo sostenible y para la ciudadanía mundial. «Tal como se establece en el cuarto Objetivo de Desarrollo Sostenible y de la Agenda 2030, la educación para el desarrollo sostenible y para la ciudadanía mundial se tendrá en cuenta en los procesos de formación del profesorado y en el acceso a la función docente. De acuerdo con lo anterior, para el año 2022 los conocimientos, habilidades y actitudes relativos a la educación para el desarrollo sostenible y para la ciudadanía mundial habrán sido incorporados al sistema de acceso a la función docente. Asimismo, en 2025 todo el personal docente deberá haber recibido cualificación en las metas establecidas en la Agenda 2030».

\section{ODS 16 y educación. A propósito del IV Plan de Acción de Gobierno Abierto.}

Siguiendo la definición dada por la OCDE, podemos definir al Gobierno Abierto como «una cultura de gobernanza que promueve los principios de transparencia, integridad, rendición de cuentas y participación de las partes interesadas en apoyo de la democracia y el crecimiento inclusivo».

España forma parte de los países integrantes de la Alianza para el Gobierno Abierto, conocida también por sus siglas en inglés OGP (Open Government Partnership), siendo ésta una iniciativa internacional surgida en 2011 cuyos objetivos principales son, entre otros, la consecución de gobiernos más transparentes, que rindan cuentas de su acción de gobierno y que promuevan una mayor participación de sus ciudadanos; una mejora de la calidad de los gobiernos, así como una reducción de los índices de corrupción. Una vez que un país es aceptado por la OGP, el siguiente paso será la redacción de un Plan de Acción de Gobierno Abierto que, en principio, está configurado para tener una vigencia bianual, aunque no siempre ha sido así. 
España, ha aprobado hasta la fecha cuatro Planes de Acción de Gobierno Abierto ${ }^{4}$, encontrándose vigente el IV Plan para el período 2020-2024, basado en cuatro ejes fundamentales: transparencia y rendición de cuentas, participación, integridad y sensibilización y formación, dentro de los cuales se insertan compromisos específicos para ser cumplidos en el período de vigencia del Plan.

La Agenda 2030 está muy presente en la práctica totalidad del IV Plan, siendo el ODS 16 «paz, justicia e instituciones sólidas» el objetivo principal que persigue cada uno de los compromisos que lo componen. No obstante, como novedad destacable de este Plan de Acción, se incluye un apartado específico que muestra la relación de cada uno de los compromisos con los ODS involucrados. Como no podía ser de otra forma, el ODS 16 está presente en todos ellos sin excepción, sin embargo, para encontrar el ODS 4 involucrado en alguno de los compromisos tenemos que acudir a las medidas de gobierno abierto adoptadas en algunas Comunidades Autónomas. En particular:

- En la Región de Murcia, con la iniciativa «fomento de la participación infantil y juvenil en el diseño de políticas públicas».

- En el Gobierno de Aragón, con la iniciativa «Openkids- programa de participación infantil».

- En el Gobierno de Navarra, con la iniciativa «Participación de niñas, niños y adolescentes».

- En la Ciudad Autónoma de Ceuta, con la iniciativa «Formación específica de empleados públicos».

- En la Ciudad Autónoma de Melilla, con la iniciativa «Formación e información sobre la participación de los jóvenes en la gestión pública».

\footnotetext{
${ }^{4}$ Un estudio comparativo sobre los distintos Planes de Acción de Gobierno Abierto aprobados por España desde su integración en la OGP en 2011, lo podemos encontrar en diversas obras de quien suscribe este artículo, en particular en: MONTERO CARO, MD., 2020. «La implementación del Gobierno Abierto en España. Elaboración y ejecución de los planes de acción» publicado en la Revista Española de la Transparencia núm. 11 y disponible en abierto en: https://revistatransparencia.com/ojs/index.php/ret/article/view/96 (fecha consulta: enero 2021).
} 
- En la Federación Española de Municipios y Provincias, con la iniciativa «Plan de consolidación e impulso de la Red de entidades locales por la transparencia y la participación ciudadana».

Como se puede vislumbrar, la participación ciudadana en general y la infantil y adolescente en particular, copan la mayor parte de compromisos e iniciativas surgidas dentro del marco del IV Plan de Acción de Gobierno Abierto. Este escenario es lógico teniendo presente la incuestionable relación entre democracia y participación ciudadana, tal y como bien refleja Castellanos al afirmar que «el siglo XXI ha hecho surgir una ciudadanía activa e informada que pide progresar hacia mejores prácticas, por lo que la participación ciudadana en las decisiones públicas adopta una importancia que no tenía en el pasado, en la medida que se anuncia como un mecanismo para perfeccionar el sistema democrático» (2020: 338).

\section{Conclusiones}

La educación es la pieza sobre la que se cimienta cualquier andamiaje democrático consistente. Es por ello especialmente significativa la incidencia que el protagonista por antonomasia de nuestro tiempo presente, el COVID-19, ha tenido en todos los órdenes vitales. La mencionada enfermedad ha incidido de manera directa en nuestro modo de vida y organización democrática y, en consecuencia, ha supuesto un torpedo en la línea de flotación que sostiene todo el entramado: el sistema educativo. La forma de contrarrestar los efectos devastadores de su paso son, inicialmente, dirigidos al orden sanitario. Pero la perspectiva temporal nos orienta hacia una política medio y largoplacista en la que sus efectos sean lo menos dañinos posibles en el ámbito educativo. Y el modo de implantar un dique de contención para ese progreso tiene como horizonte claro el cumplimiento de los ODS, especialmente el ODS 4 en materia educativa.

Es notorio que los perjuicios, especialmente a los más vulnerables, como son los niños y niñas, han sido considerables. Sin ir más lejos, además de la mengua de la calidad educativa se han producido situaciones que afectan a la esfera vital como el hecho de que muchos niños y niñas tuvieron que sufrir la pérdida de comedor escolar, 
al suspenderse las clases. Todo ello ha profundizado en las grietas de la sociedad, agravando las desigualdades. La principal en el ámbito en el que nos encontramos es la derivada del seguimiento de la enseñanza, condicionada por factores como la falta de acceso a internet o equipos informáticos. La brecha digital, en su manifestación económica, ha ampliado su radio de acción en el aspecto educativo.

El cumplimiento de los ODS, y su lema de no dejar a nadie atrás, se ha visto especialmente herido por esta penosa situación. A ello cabe agregar que no todos los niños y niñas cuentan con el mismo ambiente de estudio en casa, representación adicional de la brecha evidente derivada de la situación económica familiar. Destacamos leves intentos de paliar dicha circunstancia con algunas iniciativas que se produjeron al inicio de la situación sanitaria, como que la Televisión Pública emitiera en las primeras semanas de la pandemia programas destinados a impartir lecciones en función del curso de los niños y niñas. ${ }^{5}$

En cualquier caso el progreso de la sociedad, basado en el empuje educativo como motor del dinamismo y fuente democrática de toda organización, no puede depender de un arreglo temporal. Por ello, aunque la educación de calidad sea un ODS con entidad propia, ya hemos visto como en la educación también tienen que estar presentes de forma transversal la mayor parte de los otros 16 objetivos.

La educación es fuente y matriz de evolución democrática e inspiradora de la acción de la ciudadanía, en sus etapas de vida iniciales. Sirva como ejemplo de participación

\footnotetext{
${ }^{5}$ Destacamos a este respecto el plan de contingencia «para situaciones de emergencia» contenido en la Disposición Adicional Décima de la LOMLOE que dispone lo siguiente: «El Ministerio de Educación y Formación Profesional en colaboración con las administraciones educativas definirán un modelo de Plan de Contingencia para dar continuidad a la actividad educativa, de modo que garantice el derecho a la educación del alumnado en cualquier circunstancia. Así mismo, velarán por su desarrollo, cumplimiento y actualización en todos los centros educativos.

Este Plan incluirá aspectos organizativos y de funcionamiento del centro, la coordinación de los órganos de gobierno y de coordinación docente y las medidas que faciliten la comunicación con la comunidad educativa. Asimismo, recogerá, en su caso, la participación de los distintos miembros de la comunidad educativa en la mitigación y respuesta ante la situación de emergencia.

De igual modo, contendrá las medidas que garanticen la competencia digital del alumnado y profesorado, reduciendo en lo posible las brechas digitales de acceso y uso, y las previsiones para la revisión de los elementos del currículo y de las programaciones didácticas centradas en los aspectos más competenciales del currículo.

A tal fin el Gobierno regulará las normas necesarias para la aplicación de las medidas excepcionales y transitorias derivadas del Plan de Contingencia para situaciones de emergencia.»
} 
de la juventud el movimiento \#Fridaysforfuture, en España conocido como «viernes por el clima». Son los niños y los adolescentes los que encabezan, en ocasiones, movimientos de progreso democrático, especialmente en temas sobre los que tienen una mayor concienciación, véase el medioambiente. Ahora bien, ello es posible con un refuerzo y apuesta decidida por la educación, por lo que dar cumplimiento a los ODS en general pasa, inexorablemente, por una implantación óptima del ODS4. La adaptación, en este sentido, de la LOMLOE, más allá de la eterna disputa política en torno a la educación, resulta un paso adelante en este sentido, por lo que conviene hacer un seguimiento detallado y cercano en los próximos años de las innovaciones y mejoras en el presente y futuro de la educación en nuestro país. No se trata de una mejora departamental, sino que el progreso y, en último término, el sostenimiento de los pilares democráticos descansa en las acciones acertadas que se tomen en estos tiempos de zozobra. Solo una actividad dirigida a cumplir con los ODS, especialmente el cuarto en materia educativa, permitirá una lectura positiva de la salida de esta crisis. La pandemia pasará tarde o temprano, pero la situación educacional que hayamos fomentado en estos tiempos será determinante para presentar un futuro esperanzador y halagüeño, de progreso, o uno que haya involucionado con la amplitud de desigualdades y brechas sociales.

\section{Referencias bibliográficas}

BERASATEGUI SANCHO, N., y DARRETXE URRITXI, L., ¿Avanza la 'ley Celaá' hacia un modelo inclusivo para el alumnado de educación especial? The Conversation. Publicado el 29 de noviembre de 2020, [Consulta: 20 de diciembre de 2020]. Disponible en: https://theconversation.com/avanza-la-ley-celaa-hacia-un$\underline{\text { modelo-inclusivo-para-el-alumnado-de-educacion-especial-150985 }}$

CASTELLANOS CLARAMUNT, J., (2020). Participación ciudadana y buen gobierno democrático. Posibilidades y límites en la era digital. Madrid: Marcial Pons. 
COTINO HUESO, L., (2012). El derecho a la educación como derecho fundamental. Especial atención a su dimensión social prestacional. Madrid: Centro de Estudios Políticos y Constitucionales.

GAMBA ROMERO, A., y ARIAS CAREAGA, S. (2017). El papel de la universidad y la Cooperación Universitaria al Desarrollo en el nuevo escenario de la Agenda 2030, Revista Española de Desarrollo y Cooperación, 41, 13-24.

MARTÍNEZ USARRALDE, M.J., y LLORET CATALÁ, C. (2020). Odeseizar la educación y la cooperación para el desarrollo en España: una mirada internacional a la AOD y al ODS 4, Revista Educar, 56 (2), 333-348. DOI: https://doi.org/10.5565/rev/educar.1099

MONTERO CARO, MD., (2020). La implementación del Gobierno Abierto en España. Elaboración y ejecución de los planes de acción, Revista Española de la Transparencia, 11, 113-133.

ONU, (1948). Declaración Universal de Derechos Humanos, 10 diciembre 1948, núm. 217 A (III), [Consulta: 22 de noviembre de 2020]. Disponible en: https://www.refworld.org.es/docid/47a080e32.html.

ONU, (1960). Organización de Naciones Unidas para la Educación, la Ciencia y la Cultura (UNESCO), Convención relativa a la lucha contra las discriminaciones en la esfera de la enseñanza, 14 diciembre 1960, [Consulta: 22 de noviembre de 2020]. Disponible en: https://www.refworld.org.es/docid/5204c69f4.html

ONU, (1966). Pacto Internacional de Derechos Civiles y Políticos. Adoptado y abierto a la firma, ratificación y adhesión por la Asamblea General en su resolución 2200 A (XXI), de 16 de diciembre de 1966. Naciones Unidas, Serie de Tratados, vol. 999, p. 171, [Consulta: 22 de noviembre de 2020]. Disponible en: https://www.refworld.org.es/docid/5c92b8584.html.

ONU, (1966). Pacto Internacional de Derechos Económicos, Sociales y Culturales. Adoptado y abierto a la firma, ratificación y adhesión por la Asamblea General en su 
resolución 2200 A (XXI), de 16 de diciembre de 1966, 16 diciembre 1966, Naciones Unidas, Serie de Tratados, vol. 993, p. 3, [Consulta: 22 de noviembre de 2020]. Disponible en: https://www.refworld.org.es/docid/4c0f50bc2.html

ONU, (2015). Transformar nuestro mundo: La Agenda 2030 para el Desarrollo Sostenible. A/RES/70/1. 25 de septiembre de 2015. [Consulta: 22 de noviembre de 2020].

Disponible

en:

https://www.un.org/ga/search/view_doc.asp?symbol=A/RES/70/1\&Lang=S.

ONU, (2019). Declaración política del foro politico de alto nivel sobre el desarrollo sostenible celebrado bajo los auspicios de la Asamblea General. 24 y 25 de septiembre de 2019. [Consulta: 15 de enero de 2021]. Disponible en: https://undocs.org/pdf?symbol=es/A/HLPF/2019/1.1

ONU, (2020). Informe de los objetivos de desarrollo sostenible 2020. [Consulta: 15 de enero de 2021]. Disponible en: https://unstats.un.org/sdgs/report/2019/The$\underline{\text { Sustainable-Development-Goals-Report-2019_Spanish.pdf }}$

VICEPRESIDENCIA SEGUNDA DEL GOBIERNO. MINISTERIO DE DERECHOS SOCIALES Y AGENDA 2030, (2020). Resumen ejecutivo. Informe de Progreso 2020 sobre la implementación de la Agenda 2030 en España. Reconstruir lo común. [Consulta: 15 de enero de 2021]. Disponible en: https://www.agenda2030.gob.es/recursos/docs/21_07_Informe_Ejecutivo_ESP.pdf

VICEPRESIDENCIA SEGUNDA DEL GOBIERNO. MINISTERIO DE DERECHOS SOCIALES Y AGENDA 2030, (2021). Retos país para la Estrategia de Desarrollo Sostenible. Propuesta del grupo de trabajo de la comisión permanente del consejo de Desarrollo sostenible para la Agenda 2030. 17 de enero de 2021. [Consulta: 20 de enero de 2021]. Disponible en: https://www.agenda2030.gob.es/recursos/docs/GTEstrategia_Retos-pais_CDS.pdf

VÁZQUEZ RAMIL, R., y PORTO UCHA, Á.S., (2020). Temas transversales, ciudadanía y educación en valores: de la LOGSE (1990) a la LOMLOE (2020), Revista Innovación educativa, 30, 113-125. DOI https://doi.org/10.15304/ie.30.7092 
VIDAL PRADO, C., (2017). El derecho a la educación en España. Bases constitucionales para el acuerdo y cuestiones controvertidas. Madrid: Marcial Pons. 